\title{
RESEARCH NOTE: Some Corrections and Suggestions for Working with the National Faculty Survey Databases
}

\author{
Richard J. Bentley, Robert T. Blackburn, and Jeffery P. Bieber
}

Our ability to understand the faculty work environment has been greatly enhanced by the availability of largescale national surveys of the professoriate since the $1950 \mathrm{~s}$. This technical note identifies ways to improve data comparability among the national faculty surveys. It begins by identifying the national surveys and some of their attributes. The study then estimates faculty sizes and calculates weights for faculty in selected disciplines and institution types. The weights are designed specifically for four surveys conducted in 1969, 1975, 1980, and 1988.

Scholarly reports on U.S. faculty have a long and distinguished history. In this century, Shyrock's 1939 study on the University of Pennsylvania faculty remains a classic. So does Wilson's (1942) The Academic Man. Wilson includes a broader faculty database, although it is essentially limited to research university professors. In 1955, Lazarsfeld and Theilens (1958) conducted the first "social-scientific" national faculty survey across a wide array of institutional types, albeit only of social scientists. Parsons and Platt (1958) surveyed faculty from a wide assortment of specialties, but only in a small number of institutions. The last twenty years, however, have yielded a barrage of national surveys of faculty beginning in 1969 with more than 60,000 faculty from more than 300 institutions.

This article provides counsel to those who rely on these more recent surveys as accurate estimates of faculty perceptions, beliefs, and behaviors. It should aid those who formulate their research hypotheses or make policy recommendations

Richard J. Bentley and Robert T. Blackburn, University of Michigan. Jeffery P. Bieber, College of William and Mary. Address correspondence to: Richard Bentley, University of Michigan, 2117 SEB, Ann Arbor, MI 48109. 
regarding the future of U.S. higher education on the basis of these national surveys.

Although the national surveys have contributed considerably to our understanding of faculty, these databases contain sampling errors that can distort comparisons over time. For example, to confirm whether or not the proportion of women faculty has risen over the past two decades, one has to control for institutional type because proportionally fewer females work at research universities (Astin, 1978). While some recent national surveys proportionally stratified their samples by Carnegie type, earlier surveys have had to rely on poststratification weights to account for sampling frame biases because they oversampled research university faculty.

What confounds the matter even more is that reports disagree on how many faculty there are at different points in time, either full-time or part-time. ${ }^{1}$ For example, in 1981 the National Center for Educational Statistics (1989) says there were 462,000 full-time faculty. The American Council on Education (1987) says 470,000. Bowen and Schuster (1986) say 537,000. For 1987, NCES says there were 466,000 in one publication (1989) and 491,000 in another (OERI, 1990).

This article provides ways of improving comparability across the surveys for faculty in selected disciplines. It begins with an identification of the surveys and some of their attributes. It then provides estimates of faculty sizes and calculations for estimating weights. The weights are designed for four survey years: $1969,1975,1980$, and 1988 .

\section{THE NATIONAL SURVEYS}

Table 1 chronologically lists the national surveys by sponsor, sample size, response rate, and number of institutions involved. Explanatory notes are also provided. As indicated in Table 1, the sample populations vary considerably in size. For example, Parson and Platt sampled the smallest number of faculty $(N=420)$, while the American Council of Education surveyed the entire population of faculty $(N=108,722)$ at the 301 institutions included in their survey (Bayer, 1970, pp. 5-6).

The national surveys differ widely in questionnaire design and study objectives. ${ }^{2}$ For example, the questionnaires range from 4 pages in length (with 30 questions) for the American Council on Education survey in 1972 to 18 pages (with 87 questions) by Ladd and Lipset (1975). Even with such variation, several questions are repeated across survey years, thereby enabling comparisons over time ${ }^{3}$ (also see Drew and Tronvig, 1988).

Reliability data for the national survey questions are rare. However, the University of Michigan study found that test-retest reliabilities were good (average around .70) for the behavior variables, such as number of publications, 
TABLE 1. National Facuity Surveys

\begin{tabular}{|c|c|c|c|c|c|c|}
\hline Year & Sponsor & $\begin{array}{c}\text { Total } \\
\text { Surveys }\end{array}$ & $\begin{array}{c}\text { Total } \\
\text { Usable } \\
\text { Surveys }\end{array}$ & $\begin{array}{l}\text { Usable } \\
\text { Surveys } \\
\text { Returned }\end{array}$ & $\begin{array}{l}\text { \% Re- } \\
\text { sponse } \\
\text { Rate }\end{array}$ & $\begin{array}{l}\text { Number } \\
\text { of Insti- } \\
\text { tutions }\end{array}$ \\
\hline 1955 & Lazarsfeld \& Theilens ${ }^{1}$ & & & 2,451 & & 165 \\
\hline 1959 & Berelson $^{2}$ & 4,440 & & 1,821 & $41.0 \%$ & 92 \\
\hline 1968 & Parsons \& Platt $^{3}$ & & & 420 & & 8 \\
\hline 1969 & Carnegie/ACE ${ }^{4}$ & 100,290 & & 60,028 & 59.9 & $303^{5}$ \\
\hline 1971 & Stanford University & 17,000 & & 9,237 & 54.3 & 259 \\
\hline 1972 & $\mathrm{ACE}$ & 108,722 & & 53,131 & 48.9 & 301 \\
\hline 1975 & Ladd and Lipset ${ }^{6}$ & 7,798 & 7,253 & 3,536 & 45.3 & 111 \\
\hline 1975 & Carnegie Council & 52,876 & 47,753 & 25,262 & 47.8 & $340^{7}$ \\
\hline 1977 & Ladd \& Lipset & 8,697 & & 4,383 & 50.4 & 160 \\
\hline 1980 & UCLA & $31,302^{8}$ & 29,599 & 9,948 & 31.8 & 98 \\
\hline 1984 & Carnegie Foundation ${ }^{9}$ & 9,968 & & $5,057^{10}$ & 50.7 & 310 \\
\hline 1985 & ACLS 11 & 5,385 & & 3,835 & 71.2 & \\
\hline 1986 & $\mathrm{CIC}^{12}$ & 9,204 & & 4,271 & 46.4 & 142 \\
\hline 1988 & University of Michigan ${ }^{13}$ & 8,000 & & 3,972 & 49.7 & $236^{14}$ \\
\hline 1988 & OERI & 11,013 & & 8,383 & 76.1 & $449^{15}$ \\
\hline 1989 & Carnegie Foundation $^{16}$ & 9,996 & & 5,450 & 54.5 & 306 \\
\hline 1989 & UCLA & $93,800^{17}$ & & 51,605 & 55.0 & 432 \\
\hline
\end{tabular}

Technical Notes

${ }^{1}$ Lazarsfeld and Theilens (1958) conducted personal interviews for social science faculty only. Large public four-year universities were oversampled.

${ }^{2}$ Berelson (1960) surveyed only faculty at graduate institutions. Productivity measures include total books and articles during last five years.

${ }^{3}$ See Parsons and Platt (1968).

${ }^{4}$ Cosponsored by the Carnegie Commission on the Future of Higher Education and the American Council on Education (Bayer, 1970; Trow, 1975).

${ }^{5}$ Roizen, Fulton, and Trow (1978:8) report 307 institutions in 1969.

${ }^{6}$ According to a telephone conversation with Martin Trow, the Ladd and Lipset data were pilot data nun for the 1975 Camegie survey.

${ }^{7}$ Initially 514 institutions selected (Roizen, Fulton, and Trow, 1978).

${ }^{8}$ See discussion on response rate in Higher Education Research Institute technical report (n.d.).

${ }^{9}$ See technical report (Carnegie, 1984).

${ }^{10}$ Only $\mathrm{N}=4,999$ on tape analyzed by authors.

11 The American Council of Learned Societies selected society members from seven disciplines, including classics, history, linguistics, English and American literature, philosophy, political science, and sociology (Morton and Price, 1986).

${ }^{12}$ Council of Independent Colleges survey primarily of liberal arts II institutions.

${ }^{13}$ Includes faculty from eight disciplines: English, history, psychology, sociology, political science, biology, chemistry, and mathematics (Blackburn and Lawrence, 1989).

${ }^{14}$ Initially 250 institutions selected.

is Initially 480 institutions selected (OERI, 1990).

${ }^{16}$ See technical report (Carnegie 1989 b)

${ }^{17}$ Estimate based on $55 \%$ response rate.

but lower for perceptual and attitudinal items (Blackburn and Mackie, 1990). High reliabilities of behavior variables have also been reported by Allison and Stewart (1974), Blackburn, Boberg, O'Connell, and Pellino (1980), and Clark and Centra (1985).

\section{NUMBERS OF FACULTY AND SAMPLE WEIGHTS}

The first task in deriving multipliers to correct for survey samples is to 
determine accurate estimates of the number of full-time faculty. How these estimates were reached is explained and a table of the results is shown. Then a figure displays data by Carnegie type and control for three points in time. Tables with corrected weights are given for selected institutional types and disciplines for four survey years.

\section{The Number of Faculty}

The faculty population was defined as all full, associate, or assistant professors with teaching appointments in eight liberal arts disciplines, representing the natural sciences (biology, chemistry, mathematics), social sciences (political science, psychology, sociology), and humanities (English, history). The eight disciplines were the same as those selected by the 1988 University of Michigan survey. The Michigan survey chose these eight disciplines because they represented disciplines common to most colleges and universities and where faculty $N$ 's were expected to be highest.

The population included faculty from five of the largest Carnegie-type institutions: research I (Res-I) and research II (Res-II) universities, doctoralgranting I (Doc-I) and doctoral-granting II (Doc-II) universities, and comprehensive-I colleges and universities (Comp-I). Faculty were excluded from comprehensive-II (Comp-II), liberal arts, and two-year institutions where publication activity is less common (Bieber, 1990).

In order to estimate the number of faculty at different points in time, data were drawn from three volumes of the American Universities and College directories $(1968,1983,1987)$. Unlike other national faculty data, such as the federal Higher Education General Information Surveys (HEGIS), the directories provide detailed data broken down at the institutional level by discipline and academic rank. The three selected directories corresponded closest to the 1969, 1980 , and 1988 survey years. Because the directory had ceased publication between 1972 and 1983, the 1975 faculty estimate had to be interpolated from the 1969 and 1983 data.

\section{Sampling Technique}

To estimate mean department size, we randomly sampled institutions by Carnegie type and control from lists published by Carnegie (1973, 1976, and 1987). Eight separate samples were drawn in order to identify mean faculty department sizes by public and private control for four Carnegie categories (Res-I, Res-II, Doc-I and Doc-II combined, and Comp-I). This procedure yielded 64 cells.

As a check, we compared the estimated mean faculty department sizes with 
similar samples drawn from the same data sources in a separate, independent study (Bieber, 1990). ${ }^{4}$ Findings were comparable, with the exception of public Comp-I institutions in 1980. Further examination found that this overestimation was due to an excess sampling of larger institutions in 1980. To correct for this error, we pooled the comprehensive-I public samples from the two studies. ${ }^{5}$ Faculty estimates for the three disciplines fell within the $90 \%$ confidence interval that Bieber had determined for the same three disciplines. Since the differences of the means for these three disciplines were found to be tolerable, it is reasonable to assume that the means of the other five disciplines are also reliable. Furthermore, for those three disciplines the confidence level is well above the $90 \%$ level.

\section{Changes in Faculty by Carnegie Type}

Table 2 shows estimates of average faculty size by Carnegie type. As indicated by the data, public institutions are considerably larger than private institutions in each of the Carnegie categories. Over time, Res-II publics show the largest fluctuations, increasing from an average of 144 faculty to 237 between 1968 and 1983, while dropping back down to 196 by 1987.

Figure 1 shows that Comp-I public institutions are the largest and fastest growing employer of faculty. They have more than a third of all faculty in the target population. Between 1967 and 1985, the estimated populations of Comp-I faculty within the eight selected disciplines nearly doubled-rising from 15,833 to 29,355 (see also Table 2). The number of faculty at the other institution types remained generally constant at each point in time. There are, however, fewer faculty in public research and doctoral institutions in 1988 than there were in 1980.

\section{Weight Tables}

Tables 3 through 6 show the estimates for total number of faculty in years corresponding with the $1969,1975,1980$, and 1988 national surveys. The actual survey $N$ 's and the weights that reconcile these differences are also presented.

Faculty at Comp-I institutions were most underrepresented in both the 1969 Carnegie and the 1980 UCLA HERI surveys (see Tables 3 and 5). As a result, the weights are as high as 4.56 (Comp-I public mathematicians) in 1969 and 3.52 in 1980 (Comp-I private biologists). These higher weights reflect a sampling framework bias that oversampled selective research institutions (Bayer, 1970; Roizen, Fulton, and Trow, 1978). Also, because the 1980 HERI 


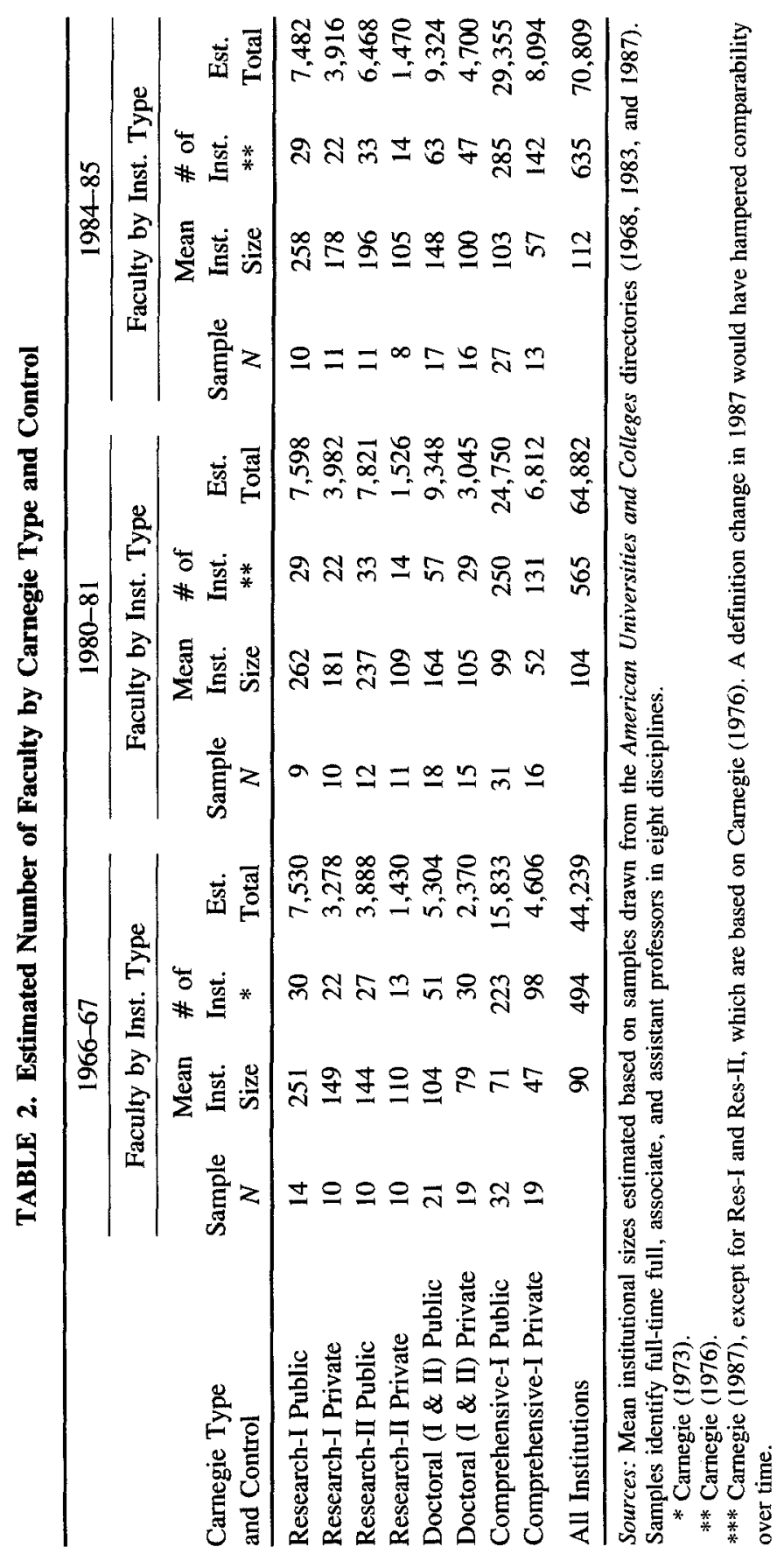




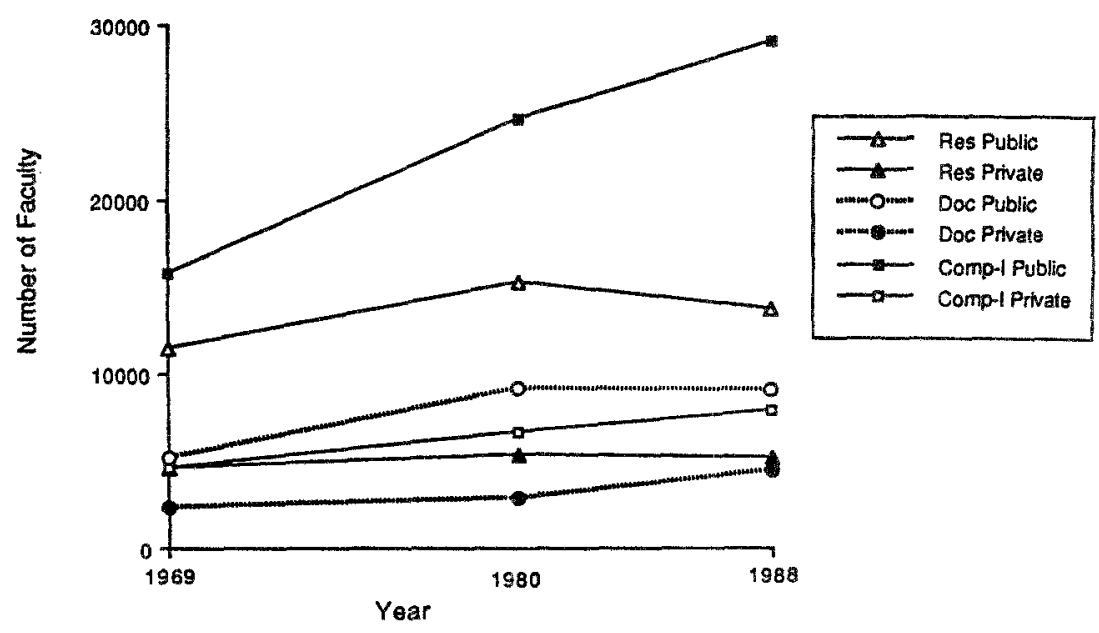

FIG. 1. Changes in faculty by Camegie type and control.

study replicated the 1969 sampling framework (HERI, n.d.), it too had a lower proportional representation of Comp-I faculty.

By contrast, the 1975 Carnegie and 1988 University of Michigan samples more accurately reflected the faculty population estimates. As a result, the weights were less extreme (see Tables 4 and 6). For example, all the 1975 and 1988 weights were less than 2, with the exception of public Res-II mathematicians in 1988, which had a weight of 3.55. The more accurate representation of the 1975 survey makes sense since Trow, who also worked on the 1969 Carnegie survey, expanded the 1975 sampling framework to include faculty that had been undersampled in 1969 and 1972. Trow added faculty at less selective four-year colleges and historically black institutions (Roizen, Fulton, and Trow, 1978). Meanwhile, the 1988 weights were less extreme because the University of Michigan study sampled faculty in proportion to their numbers in Carnegie types (Blackburn and Lawrence, 1989).

\section{CONCLUSION}

Our ability to understand the faculty work environment has been greatly enhanced by the availability of largescale national surveys of the professoriate. Since the mid-1950s, organizations have sponsored at least 17 national surveys that examine a variety of faculty behaviors and attitudes. Moreover, interest in faculty appears to be waxing as the number of surveys has increased in recent years (4 since 1987).

Although many surveys have intentionally replicated questions from earlier surveys, the wide fluctuation of weights presented in this paper illustrates the 
TABLE 3. 1969 Carnegie Weights

\begin{tabular}{|c|c|c|c|c|c|c|c|c|}
\hline \multirow{2}{*}{$\begin{array}{l}\text { Carnegie } \\
\text { Type }\end{array}$} & \multirow[b]{2}{*}{ Discipline } & \multicolumn{2}{|c|}{$\begin{array}{c}\text { Estimated } \\
1969 \text { Faculty }\end{array}$} & \multicolumn{2}{|c|}{$\begin{array}{l}1969 \text { Carnegie } \\
\text { Subsample }\end{array}$} & \multirow[b]{2}{*}{ Weight } & \multicolumn{2}{|c|}{$\begin{array}{c}\text { Weighted } \\
1969 \text { Subsample }\end{array}$} \\
\hline & & $N$ & $\%$ & $N$ & $\%$ & & $N$ & $\%$ \\
\hline $\begin{array}{l}\text { Res-I } \\
\text { Public }\end{array}$ & $\begin{array}{l}\text { bio } \\
\text { chem } \\
\text { Eng } \\
\text { hist } \\
\text { math } \\
\text { polsci } \\
\text { soc } \\
\text { psych }\end{array}$ & $\begin{array}{r}600 \\
990 \\
1560 \\
810 \\
1470 \\
600 \\
450 \\
1050\end{array}$ & $\begin{array}{l}1.4 \% \\
2.2 \% \\
3.5 \% \\
1.8 \% \\
3.3 \% \\
1.4 \% \\
1.0 \% \\
2.4 \%\end{array}$ & $\begin{array}{r}31 \\
119 \\
194 \\
127 \\
201 \\
83 \\
78 \\
166\end{array}$ & $\begin{array}{l}1.0 \% \\
3.8 \% \\
6.1 \% \\
4.0 \% \\
6.3 \% \\
2.6 \% \\
2.5 \% \\
5.2 \%\end{array}$ & $\begin{array}{l}1.39 \\
0.60 \\
0.58 \\
0.46 \\
0.52 \\
0.52 \\
0.41 \\
0.45\end{array}$ & $\begin{array}{r}43 \\
71 \\
112 \\
58 \\
105 \\
43 \\
32 \\
75\end{array}$ & $\begin{array}{l}1.4 \% \\
2.2 \% \\
3.5 \% \\
1.8 \% \\
3.3 \% \\
1.4 \% \\
1.0 \% \\
2.4 \%\end{array}$ \\
\hline $\begin{array}{l}\text { Res-I } \\
\text { Private }\end{array}$ & $\begin{array}{l}\text { bio } \\
\text { chem } \\
\text { Eng } \\
\text { hist } \\
\text { math } \\
\text { polsci } \\
\text { soc } \\
\text { psych }\end{array}$ & $\begin{array}{l}374 \\
506 \\
550 \\
462 \\
550 \\
308 \\
220 \\
308\end{array}$ & $\begin{array}{l}0.8 \% \\
1.1 \% \\
1.2 \% \\
1.0 \% \\
1.2 \% \\
0.7 \% \\
0.5 \% \\
0.7 \%\end{array}$ & $\begin{array}{l}45 \\
62 \\
54 \\
67 \\
75 \\
43 \\
37 \\
50\end{array}$ & $\begin{array}{l}1.4 \% \\
2.0 \% \\
1.7 \% \\
2.1 \% \\
2.4 \% \\
1.4 \% \\
1.2 \% \\
1.6 \%\end{array}$ & $\begin{array}{l}0.59 \\
0.58 \\
0.73 \\
0.49 \\
0.52 \\
0.51 \\
0.43 \\
0.44\end{array}$ & $\begin{array}{l}27 \\
36 \\
39 \\
33 \\
39 \\
22 \\
16 \\
22\end{array}$ & $\begin{array}{l}0.8 \% \\
1.1 \% \\
1.2 \% \\
1.0 \% \\
1.2 \% \\
0.7 \% \\
0.5 \% \\
0.7 \%\end{array}$ \\
\hline $\begin{array}{l}\text { Res-II } \\
\text { Public }\end{array}$ & $\begin{array}{l}\text { bio } \\
\text { chem } \\
\text { Eng } \\
\text { hist } \\
\text { math } \\
\text { polsci } \\
\text { soc } \\
\text { psych }\end{array}$ & $\begin{array}{l}405 \\
594 \\
810 \\
459 \\
621 \\
351 \\
270 \\
378\end{array}$ & $\begin{array}{l}0.9 \% \\
1.3 \% \\
1.8 \% \\
1.0 \% \\
1.4 \% \\
0.8 \% \\
0.6 \% \\
0.9 \%\end{array}$ & $\begin{array}{l}12 \\
70 \\
84 \\
43 \\
77 \\
39 \\
25 \\
40\end{array}$ & $\begin{array}{l}0.4 \% \\
2.2 \% \\
2.7 \% \\
1.4 \% \\
2.4 \% \\
1.2 \% \\
0.8 \% \\
1.3 \%\end{array}$ & $\begin{array}{l}2.42 \\
0.61 \\
0.69 \\
0.76 \\
0.58 \\
0.64 \\
0.77 \\
0.68\end{array}$ & $\begin{array}{l}29 \\
43 \\
58 \\
33 \\
44 \\
25 \\
19 \\
27\end{array}$ & $\begin{array}{l}0.9 \% \\
1.3 \% \\
1.8 \% \\
1.0 \% \\
1.4 \% \\
0.8 \% \\
0.6 \% \\
0.9 \%\end{array}$ \\
\hline $\begin{array}{l}\text { Res-II } \\
\text { Private }\end{array}$ & $\begin{array}{l}\text { bio } \\
\text { chem } \\
\text { Eng } \\
\text { hist } \\
\text { math } \\
\text { polsci } \\
\text { soc } \\
\text { psych }\end{array}$ & $\begin{array}{l}208 \\
195 \\
273 \\
169 \\
195 \\
117 \\
104 \\
169\end{array}$ & $\begin{array}{l}0.5 \% \\
0.4 \% \\
0.6 \% \\
0.4 \% \\
0.4 \% \\
0.3 \% \\
0.2 \% \\
0.4 \%\end{array}$ & $\begin{array}{r}12 \\
11 \\
16 \\
12 \\
11 \\
9 \\
8 \\
10\end{array}$ & $\begin{array}{l}0.4 \% \\
0.3 \% \\
0.5 \% \\
0.4 \% \\
0.3 \% \\
0.3 \% \\
0.3 \% \\
0.3 \%\end{array}$ & $\begin{array}{l}1.24 \\
1.27 \\
1.22 \\
1.01 \\
1.27 \\
0.93 \\
0.93 \\
1.21\end{array}$ & $\begin{array}{r}15 \\
14 \\
20 \\
12 \\
14 \\
8 \\
7 \\
12\end{array}$ & $\begin{array}{l}0.5 \% \\
0.4 \% \\
0.6 \% \\
0.4 \% \\
0.4 \% \\
0.3 \% \\
0.2 \% \\
0.4 \%\end{array}$ \\
\hline $\begin{array}{l}\text { Doc-I } \\
\& \\
\text { Doc-II } \\
\text { Public }\end{array}$ & $\begin{array}{l}\text { bio } \\
\text { chem } \\
\text { Eng } \\
\text { hist } \\
\text { math } \\
\text { polsci } \\
\text { soc } \\
\text { psych }\end{array}$ & $\begin{array}{r}663 \\
612 \\
1224 \\
714 \\
765 \\
408 \\
306 \\
612\end{array}$ & $\begin{array}{l}1.5 \% \\
1.4 \% \\
2.8 \% \\
1.6 \% \\
1.7 \% \\
0.9 \% \\
0.7 \% \\
1.4 \%\end{array}$ & $\begin{array}{r}54 \\
93 \\
103 \\
76 \\
82 \\
55 \\
45 \\
74\end{array}$ & $\begin{array}{l}1.7 \% \\
2.9 \% \\
3.3 \% \\
2.4 \% \\
2.6 \% \\
1.7 \% \\
1.4 \% \\
2.3 \%\end{array}$ & $\begin{array}{l}0.88 \\
0.47 \\
0.85 \\
0.67 \\
0.67 \\
0.53 \\
0.49 \\
0.59\end{array}$ & $\begin{array}{l}47 \\
44 \\
88 \\
51 \\
55 \\
29 \\
22 \\
44\end{array}$ & $\begin{array}{l}1.5 \% \\
1.4 \% \\
2.8 \% \\
1.6 \% \\
1.7 \% \\
0.9 \% \\
0.7 \% \\
1.4 \%\end{array}$ \\
\hline
\end{tabular}


TABLE 3. (Continued)

\begin{tabular}{|c|c|c|c|c|c|c|c|c|}
\hline \multirow{2}{*}{$\begin{array}{l}\text { Carnegie } \\
\text { Type }\end{array}$} & \multirow[b]{2}{*}{ Discipline } & \multicolumn{2}{|c|}{$\begin{array}{c}\text { Estimated } \\
1969 \text { Faculty }\end{array}$} & \multicolumn{2}{|c|}{$\begin{array}{c}1969 \text { Carnegie } \\
\text { Subsample }\end{array}$} & \multirow[b]{2}{*}{ Weight } & \multicolumn{2}{|c|}{$\begin{array}{c}\text { Weighted } \\
1969 \text { Subsample }\end{array}$} \\
\hline & & $N$ & $\%$ & $N$ & $\%$ & & $N$ & $\%$ \\
\hline Doc-I & bio & 270 & $0.6 \%$ & 31 & $1.0 \%$ & 0.62 & 19 & $0.6 \%$ \\
\hline$\&$ & chem & 300 & $0.7 \%$ & 28 & $0.9 \%$ & 0.77 & 21 & $0.7 \%$ \\
\hline Doc-II & Eng & 450 & $1.0 \%$ & 36 & $1.1 \%$ & 0.89 & 32 & $1.0 \%$ \\
\hline \multirow[t]{5}{*}{ Private } & hist & 360 & $0.8 \%$ & 28 & $0.9 \%$ & 0.92 & 26 & $0.8 \%$ \\
\hline & math & 330 & $0.7 \%$ & 38 & $1.2 \%$ & 0.62 & 24 & $0.7 \%$ \\
\hline & polsci & 210 & $0.5 \%$ & 22 & $0.7 \%$ & 0.68 & 15 & $0.5 \%$ \\
\hline & soc & 150 & $0.3 \%$ & 14 & $0.4 \%$ & 0.77 & 11 & $0.3 \%$ \\
\hline & psych & 300 & $0.7 \%$ & 38 & $1.2 \%$ & 0.56 & 21 & $0.7 \%$ \\
\hline Comp-I & bio & 2230 & $5.0 \%$ & 48 & $1.5 \%$ & 3.32 & 160 & $5.0 \%$ \\
\hline \multirow{7}{*}{ Public } & chem & 1561 & $3.5 \%$ & 46 & $1.5 \%$ & 2.43 & 112 & $3.5 \%$ \\
\hline & Eng & 3568 & $8.1 \%$ & 62 & $2.0 \%$ & 4.12 & 255 & $8.1 \%$ \\
\hline & hist & 2007 & $4.5 \%$ & 42 & $1.3 \%$ & 3.42 & 144 & $4.5 \%$ \\
\hline & math & 2230 & $5.0 \%$ & 35 & $1.1 \%$ & 4.56 & 160 & $5.0 \%$ \\
\hline & polsci & 1338 & $3.0 \%$ & 22 & $0.7 \%$ & 4.35 & 96 & $3.0 \%$ \\
\hline & soc & 1115 & $2.5 \%$ & 20 & $0.6 \%$ & 3.99 & 80 & $2.5 \%$ \\
\hline & psych & 1784 & $4.0 \%$ & 40 & $1.3 \%$ & 3.19 & 128 & $4.0 \%$ \\
\hline Comp-I & bio & 588 & $1.3 \%$ & 11 & $0.3 \%$ & 3.83 & 42 & $1.3 \%$ \\
\hline \multirow{9}{*}{ Private } & chem & 588 & $1.3 \%$ & 16 & $0.5 \%$ & 2.63 & 42 & $1.3 \%$ \\
\hline & Eng & 1078 & $2.4 \%$ & 27 & $0.9 \%$ & 2.86 & 77 & $2.4 \%$ \\
\hline & hist & 686 & $1.6 \%$ & 19 & $0.6 \%$ & 2.58 & 49 & $1.6 \%$ \\
\hline & math & 588 & $1.3 \%$ & 18 & $0.6 \%$ & 2.34 & 42 & $1.3 \%$ \\
\hline & polsci & 294 & $0.7 \%$ & 10 & $0.3 \%$ & 2.10 & 21 & $0.7 \%$ \\
\hline & soc & 294 & $0.7 \%$ & 5 & $0.2 \%$ & 4.21 & 21 & $0.7 \%$ \\
\hline & psych & 490 & $1.1 \%$ & 17 & $0.5 \%$ & 2.06 & 35 & $1.1 \%$ \\
\hline & Grand & & & & & & & \\
\hline & Total & 44239 & $100 \%$ & 3166 & $100 \%$ & & 3166 & $100 \%$ \\
\hline
\end{tabular}

Sources: Estimated faculty based on 1966-67 data reported in the American Universities and Colleges directory (1968).

extent to which unweighted samples can distort data comparisons over time. To adjust for such distortions resulting from differences in sampling populations, response rates, and poststratification weighting methods, we created weights to reflect systematically changes in the number of the faculty by discipline, Carnegie institution type, and institutional control. The weights were based on data drawn from the consistent outside data source corresponding in time with four of the national surveys.

Comparability of data over time may be especially susceptible to fluctuations in the composition of faculty by disciplinary or institutional type since studies have found that faculty behaviors differ significantly by disciplines (Biglan, 
TABLE 4. 1975 Carnegie Weights

\begin{tabular}{|c|c|c|c|c|c|c|c|c|}
\hline \multirow{2}{*}{$\begin{array}{l}\text { Carnegie } \\
\text { Type }\end{array}$} & \multirow[b]{2}{*}{ Discipline } & \multicolumn{2}{|c|}{$\begin{array}{c}\text { Estimated } \\
1975 \text { Faculty }\end{array}$} & \multicolumn{2}{|c|}{$\begin{array}{l}1975 \text { Carnegie } \\
\text { Subsample }\end{array}$} & \multirow[b]{2}{*}{ Weight } & \multicolumn{2}{|c|}{$\begin{array}{c}\text { Weighted } \\
1975 \text { Subsample }\end{array}$} \\
\hline & & $N$ & $\%$ & $N$ & $\%$ & & $N$ & $\%$ \\
\hline $\begin{array}{l}\text { Res-I } \\
\text { Public }\end{array}$ & $\begin{array}{l}\text { bio } \\
\text { chem } \\
\text { Eng } \\
\text { hist } \\
\text { math } \\
\text { polsci } \\
\text { soc } \\
\text { psych }\end{array}$ & $\begin{array}{r}679 \\
924 \\
1392 \\
882 \\
1355 \\
630 \\
567 \\
1032\end{array}$ & $\begin{array}{l}1.2 \% \\
1.6 \% \\
2.4 \% \\
1.5 \% \\
2.3 \% \\
1.1 \% \\
1.0 \% \\
1.8 \%\end{array}$ & $\begin{array}{r}27 \\
72 \\
105 \\
80 \\
101 \\
57 \\
38 \\
74\end{array}$ & $\begin{array}{l}0.8 \% \\
2.0 \% \\
3.0 \% \\
2.3 \% \\
2.9 \% \\
1.6 \% \\
1.1 \% \\
2.1 \%\end{array}$ & $\begin{array}{l}1.53 \\
0.78 \\
0.80 \\
0.67 \\
0.81 \\
0.67 \\
0.91 \\
0.85\end{array}$ & $\begin{array}{l}41 \\
56 \\
85 \\
54 \\
82 \\
38 \\
34 \\
63\end{array}$ & $\begin{array}{l}1.2 \% \\
1.6 \% \\
2.4 \% \\
1.5 \% \\
2.3 \% \\
1.1 \% \\
1.0 \% \\
1.8 \%\end{array}$ \\
\hline $\begin{array}{l}\text { Res-I } \\
\text { Private }\end{array}$ & $\begin{array}{l}\text { bio } \\
\text { chem } \\
\text { Eng } \\
\text { hist } \\
\text { math } \\
\text { polsci } \\
\text { soc } \\
\text { psych }\end{array}$ & $\begin{array}{l}437 \\
481 \\
625 \\
512 \\
588 \\
358 \\
270 \\
408\end{array}$ & $\begin{array}{l}0.8 \% \\
0.8 \% \\
1.1 \% \\
0.9 \% \\
1.0 \% \\
0.6 \% \\
0.5 \% \\
0.7 \%\end{array}$ & $\begin{array}{l}38 \\
59 \\
63 \\
58 \\
80 \\
55 \\
41 \\
46\end{array}$ & $\begin{array}{l}1.1 \% \\
1.7 \% \\
1.8 \% \\
1.6 \% \\
2.3 \% \\
1.6 \% \\
1.2 \% \\
1.3 \%\end{array}$ & $\begin{array}{l}0.70 \\
0.49 \\
0.60 \\
0.54 \\
0.45 \\
0.40 \\
0.40 \\
0.54\end{array}$ & $\begin{array}{l}27 \\
29 \\
38 \\
31 \\
36 \\
22 \\
16 \\
25\end{array}$ & $\begin{array}{l}0.8 \% \\
0.8 \% \\
1.1 \% \\
0.9 \% \\
1.0 \% \\
0.6 \% \\
0.5 \% \\
0.7 \%\end{array}$ \\
\hline $\begin{array}{l}\text { Res-II } \\
\text { Public }\end{array}$ & $\begin{array}{l}\text { bio } \\
\text { chem } \\
\text { Eng } \\
\text { hist } \\
\text { math } \\
\text { polsci } \\
\text { soc } \\
\text { psych }\end{array}$ & $\begin{array}{r}702 \\
801 \\
1310 \\
787 \\
1041 \\
561 \\
518 \\
782\end{array}$ & $\begin{array}{l}1.2 \% \\
1.4 \% \\
2.3 \% \\
1.4 \% \\
1.8 \% \\
1.0 \% \\
0.9 \% \\
1.3 \%\end{array}$ & $\begin{array}{l}23 \\
36 \\
71 \\
46 \\
52 \\
32 \\
34 \\
28\end{array}$ & $\begin{array}{l}0.7 \% \\
1.0 \% \\
2.0 \% \\
1.3 \% \\
1.5 \% \\
0.9 \% \\
1.0 \% \\
0.8 \%\end{array}$ & $\begin{array}{l}1.85 \\
1.35 \\
1.12 \\
1.04 \\
1.22 \\
1.06 \\
0.92 \\
1.69\end{array}$ & $\begin{array}{l}43 \\
49 \\
79 \\
48 \\
63 \\
34 \\
31 \\
47\end{array}$ & $\begin{array}{l}1.2 \% \\
1.4 \% \\
2.3 \% \\
1.4 \% \\
1.8 \% \\
1.0 \% \\
0.9 \% \\
1.3 \%\end{array}$ \\
\hline $\begin{array}{l}\text { Res-II } \\
\text { Private }\end{array}$ & $\begin{array}{l}\text { bio } \\
\text { chem } \\
\text { Eng } \\
\text { hist } \\
\text { math } \\
\text { polsci } \\
\text { soc } \\
\text { psych }\end{array}$ & $\begin{array}{l}192 \\
194 \\
254 \\
198 \\
218 \\
150 \\
112 \\
214\end{array}$ & $\begin{array}{l}0.3 \% \\
0.3 \% \\
0.4 \% \\
0.3 \% \\
0.4 \% \\
0.3 \% \\
0.2 \% \\
0.4 \%\end{array}$ & $\begin{array}{l}35 \\
45 \\
41 \\
39 \\
46 \\
30 \\
17 \\
27\end{array}$ & $\begin{array}{l}1.0 \% \\
1.3 \% \\
1.2 \% \\
1.1 \% \\
1.3 \% \\
0.9 \% \\
0.5 \% \\
0.8 \%\end{array}$ & $\begin{array}{l}0.33 \\
0.26 \\
0.38 \\
0.31 \\
0.29 \\
0.30 \\
0.40 \\
0.48\end{array}$ & $\begin{array}{r}12 \\
12 \\
15 \\
12 \\
13 \\
9 \\
7 \\
13\end{array}$ & $\begin{array}{l}0.3 \% \\
0.3 \% \\
0.4 \% \\
0.3 \% \\
0.4 \% \\
0.3 \% \\
0.2 \% \\
0.4 \%\end{array}$ \\
\hline $\begin{array}{l}\text { Doc-I } \\
\& \\
\text { Doc-II } \\
\text { Public }\end{array}$ & $\begin{array}{l}\text { bio } \\
\text { chem } \\
\text { Eng } \\
\text { hist } \\
\text { math } \\
\text { polsci } \\
\text { soc } \\
\text { psych }\end{array}$ & $\begin{array}{r}1001 \\
814 \\
1628 \\
928 \\
1180 \\
683 \\
602 \\
1041\end{array}$ & $\begin{array}{l}1.7 \% \\
1.4 \% \\
2.8 \% \\
1.6 \% \\
2.0 \% \\
1.2 \% \\
1.0 \% \\
1.8 \%\end{array}$ & $\begin{array}{l}40 \\
62 \\
99 \\
58 \\
63 \\
49 \\
32 \\
43\end{array}$ & $\begin{array}{l}1.1 \% \\
1.8 \% \\
2.8 \% \\
1.6 \% \\
1.8 \% \\
1.4 \% \\
0.9 \% \\
1.2 \%\end{array}$ & $\begin{array}{l}1.52 \\
0.80 \\
1.00 \\
0.97 \\
1.14 \\
0.85 \\
1.14 \\
1.47\end{array}$ & $\begin{array}{l}61 \\
49 \\
99 \\
56 \\
72 \\
41 \\
37 \\
63\end{array}$ & $\begin{array}{l}1.7 \% \\
1.4 \% \\
2.8 \% \\
1.6 \% \\
2.0 \% \\
1.2 \% \\
1.0 \% \\
1.8 \% \\
\text { intinued) }\end{array}$ \\
\hline
\end{tabular}


TABLE 4. (Continued)

\begin{tabular}{|c|c|c|c|c|c|c|c|c|}
\hline \multirow{2}{*}{$\begin{array}{l}\text { Carnegie } \\
\text { Type }\end{array}$} & \multirow[b]{2}{*}{ Discipline } & \multicolumn{2}{|c|}{$\begin{array}{c}\text { Estimated } \\
1975 \text { Faculty }\end{array}$} & \multicolumn{2}{|c|}{$\begin{array}{c}1975 \text { Carnegie } \\
\text { Subsample }\end{array}$} & \multirow[b]{2}{*}{ Weight } & \multicolumn{2}{|c|}{$\begin{array}{c}\text { Weighted } \\
1975 \text { Subsample }\end{array}$} \\
\hline & & $N$ & $\%$ & $N$ & $\%$ & & $N$ & $\%$ \\
\hline Doc-I & bio & 311 & $0.5 \%$ & 18 & $0.5 \%$ & 1.05 & 19 & $0.5 \%$ \\
\hline$\&$ & chem & 340 & $0.6 \%$ & 35 & $1.0 \%$ & 0.59 & 21 & $0.6 \%$ \\
\hline Doc-II & Eng & 518 & $0.9 \%$ & 45 & $1.3 \%$ & 0.70 & 31 & $0.9 \%$ \\
\hline \multirow[t]{5}{*}{ Private } & hist & 331 & $0.6 \%$ & 31 & $0.9 \%$ & 0.65 & 20 & $0.6 \%$ \\
\hline & math & 402 & $0.7 \%$ & 29 & $0.8 \%$ & 0.84 & 24 & $0.7 \%$ \\
\hline & polsci & 269 & $0.5 \%$ & 22 & $0.6 \%$ & 0.74 & 16 & $0.5 \%$ \\
\hline & soc & 211 & $0.4 \%$ & 18 & $0.5 \%$ & 0.71 & 13 & $0.4 \%$ \\
\hline & psych & 340 & $0.6 \%$ & 32 & $0.9 \%$ & 0.64 & 21 & $0.6 \%$ \\
\hline Comp-I & bio & 3070 & $5.3 \%$ & 112 & $3.2 \%$ & 1.66 & 186 & $5.3 \%$ \\
\hline \multirow[t]{7}{*}{ Public } & chem & 2035 & $3.5 \%$ & 102 & $2.9 \%$ & 1.21 & 124 & $3.5 \%$ \\
\hline & Eng & 4713 & $8.1 \%$ & 208 & $5.9 \%$ & 1.38 & 286 & $8.1 \%$ \\
\hline & hist & 2677 & $4.6 \%$ & 130 & $3.7 \%$ & 1.25 & 163 & $4.6 \%$ \\
\hline & math & 3070 & $5.3 \%$ & 136 & $3.9 \%$ & 1.37 & 186 & $5.3 \%$ \\
\hline & polsci & 1643 & $2.8 \%$ & 60 & $1.7 \%$ & 1.66 & 100 & $2.8 \%$ \\
\hline & soc & 1678 & $2.9 \%$ & 82 & $2.3 \%$ & 1.24 & 102 & $2.9 \%$ \\
\hline & psych & 2855 & $4.9 \%$ & 109 & $3.1 \%$ & 1.59 & 173 & $4.9 \%$ \\
\hline Comp-I & bio & 861 & $1.5 \%$ & 47 & $1.3 \%$ & 1.11 & 52 & $1.5 \%$ \\
\hline \multirow[t]{9}{*}{ Private } & chem & 786 & $1.4 \%$ & 38 & $1.1 \%$ & 1.26 & 48 & $1.4 \%$ \\
\hline & Eng & 1441 & $2.5 \%$ & 61 & $1.7 \%$ & 1.43 & 87 & $2.5 \%$ \\
\hline & hist & 842 & $1.5 \%$ & 41 & $1.2 \%$ & 1.25 & 51 & $1.5 \%$ \\
\hline & math & 935 & $1.6 \%$ & 49 & $1.4 \%$ & 1.16 & 57 & $1.6 \%$ \\
\hline & polsci & 468 & $0.8 \%$ & 25 & $0.7 \%$ & 1.14 & 28 & $0.8 \%$ \\
\hline & soc & 468 & $0.8 \%$ & 21 & $0.6 \%$ & 1.35 & 28 & $0.8 \%$ \\
\hline & psych & 730 & $1.3 \%$ & 30 & $0.9 \%$ & 1.48 & 44 & $1.3 \%$ \\
\hline & Grand & & & & & & & \\
\hline & Total & 58042 & $100 \%$ & 3523 & $100 \%$ & & 3523 & $100 \%$ \\
\hline
\end{tabular}

Sources: Estimated faculty based on interpolation of 1966-67 and 1982-83 data reported in American Universities and Colleges directory $(1968,1983)$.

1973; Wanner, Lewis, and Gregorio, 1981) and institutional type (Long, 1978; Long and McGinnis, 1981). To adjust for such changes, the weights targeted a specific population of faculty representing eight liberal arts disciplines at larger four-year institutions. Excluded were faculty from other disciplines, such as professional fields (e.g., business, engineering) and smaller institutions (e.g., Comp-II and liberal arts colleges). ${ }^{6}$ However, researchers interested in expanding the weights to include other disciplines or types of four-year institutions could do so because the American Universities and Colleges directories include such data. 
TABLE 5. 1980 UCLA Weights

\begin{tabular}{|c|c|c|c|c|c|c|c|c|}
\hline \multirow{2}{*}{$\begin{array}{l}\text { Carnegie } \\
\text { Type }\end{array}$} & \multirow[b]{2}{*}{ Discipline } & \multicolumn{2}{|c|}{$\begin{array}{c}\text { Estimated } \\
1980 \text { Faculty }\end{array}$} & \multicolumn{2}{|c|}{$\begin{array}{l}1980 \text { UCLA } \\
\text { Subsample }\end{array}$} & \multirow[b]{2}{*}{ Weight } & \multicolumn{2}{|c|}{$\begin{array}{c}\text { Weighted } \\
1980 \text { Subsample }\end{array}$} \\
\hline & & $N$ & $\%$ & $N$ & $\%$ & & $N$ & $\%$ \\
\hline $\begin{array}{l}\text { Res-I } \\
\text { Public }\end{array}$ & $\begin{array}{l}\text { bio } \\
\text { chem } \\
\text { Eng } \\
\text { hist } \\
\text { math } \\
\text { polsci } \\
\text { soc } \\
\text { psych }\end{array}$ & $\begin{array}{r}754 \\
899 \\
1305 \\
957 \\
1305 \\
667 \\
667 \\
1044\end{array}$ & $\begin{array}{l}1.2 \% \\
1.4 \% \\
2.0 \% \\
1.5 \% \\
2.0 \% \\
1.0 \% \\
1.0 \% \\
1.6 \%\end{array}$ & $\begin{array}{l}14 \\
39 \\
31 \\
32 \\
46 \\
22 \\
23 \\
29\end{array}$ & $\begin{array}{l}0.9 \% \\
2.6 \% \\
2.1 \% \\
2.1 \% \\
3.1 \% \\
1.5 \% \\
1.5 \% \\
1.9 \%\end{array}$ & $\begin{array}{l}1.24 \\
0.53 \\
0.97 \\
0.69 \\
0.65 \\
0.70 \\
0.67 \\
0.83\end{array}$ & $\begin{array}{l}17 \\
21 \\
30 \\
22 \\
30 \\
15 \\
15 \\
24\end{array}$ & $\begin{array}{l}1.2 \% \\
1.4 \% \\
2.0 \% \\
1.5 \% \\
2.0 \% \\
1.0 \% \\
1.0 \% \\
1.6 \%\end{array}$ \\
\hline $\begin{array}{l}\text { Res-I } \\
\text { Private }\end{array}$ & $\begin{array}{l}\text { bio } \\
\text { chem } \\
\text { Eng } \\
\text { hist } \\
\text { math } \\
\text { polsci } \\
\text { soc } \\
\text { psych }\end{array}$ & $\begin{array}{l}484 \\
462 \\
682 \\
550 \\
616 \\
396 \\
308 \\
484\end{array}$ & $\begin{array}{l}0.7 \% \\
0.7 \% \\
1.1 \% \\
0.8 \% \\
0.9 \% \\
0.6 \% \\
0.5 \% \\
0.7 \%\end{array}$ & $\begin{array}{l}20 \\
15 \\
22 \\
23 \\
27 \\
25 \\
22 \\
19\end{array}$ & $\begin{array}{l}1.3 \% \\
1.0 \% \\
1.5 \% \\
1.5 \% \\
1.8 \% \\
1.7 \% \\
1.5 \% \\
1.3 \%\end{array}$ & $\begin{array}{l}0.56 \\
0.71 \\
0.71 \\
0.55 \\
0.52 \\
0.36 \\
0.32 \\
0.59\end{array}$ & $\begin{array}{r}11 \\
11 \\
16 \\
13 \\
14 \\
9 \\
7 \\
11\end{array}$ & $\begin{array}{l}0.7 \% \\
0.7 \% \\
1.1 \% \\
0.8 \% \\
0.9 \% \\
0.6 \% \\
0.5 \% \\
0.7 \%\end{array}$ \\
\hline $\begin{array}{l}\text { Res-II } \\
\text { Public }\end{array}$ & $\begin{array}{l}\text { bio } \\
\text { chem } \\
\text { Eng } \\
\text { hist } \\
\text { math } \\
\text { polsci } \\
\text { soc } \\
\text { psych }\end{array}$ & $\begin{array}{r}858 \\
858 \\
1551 \\
957 \\
1254 \\
660 \\
660 \\
1023\end{array}$ & $\begin{array}{l}1.3 \% \\
1.3 \% \\
2.4 \% \\
1.5 \% \\
1.9 \% \\
1.0 \% \\
1.0 \% \\
1.6 \%\end{array}$ & $\begin{array}{l}10 \\
24 \\
31 \\
25 \\
31 \\
19 \\
22 \\
35\end{array}$ & $\begin{array}{l}0.7 \% \\
1.6 \% \\
2.1 \% \\
1.7 \% \\
2.1 \% \\
1.3 \% \\
1.5 \% \\
2.3 \%\end{array}$ & $\begin{array}{l}1.97 \\
0.82 \\
1.15 \\
0.88 \\
0.93 \\
0.80 \\
0.69 \\
0.67\end{array}$ & $\begin{array}{l}20 \\
20 \\
36 \\
22 \\
29 \\
15 \\
15 \\
24\end{array}$ & $\begin{array}{l}1.3 \% \\
1.3 \% \\
2.4 \% \\
1.5 \% \\
1.9 \% \\
1.0 \% \\
1.0 \% \\
1.6 \%\end{array}$ \\
\hline $\begin{array}{l}\text { Res-II } \\
\text { Private }\end{array}$ & $\begin{array}{l}\text { bio } \\
\text { chem } \\
\text { Eng } \\
\text { hist } \\
\text { math } \\
\text { polsci } \\
\text { soc } \\
\text { psych }\end{array}$ & $\begin{array}{l}168 \\
182 \\
224 \\
210 \\
224 \\
168 \\
112 \\
238\end{array}$ & $\begin{array}{l}0.3 \% \\
0.3 \% \\
0.3 \% \\
0.3 \% \\
0.3 \% \\
0.3 \% \\
0.2 \% \\
0.4 \%\end{array}$ & $\begin{array}{r}6 \\
12 \\
10 \\
9 \\
9 \\
4 \\
6 \\
11\end{array}$ & $\begin{array}{l}0.4 \% \\
0.8 \% \\
0.7 \% \\
0.6 \% \\
0.6 \% \\
0.3 \% \\
0.4 \% \\
0.7 \%\end{array}$ & $\begin{array}{l}0.64 \\
0.35 \\
0.52 \\
0.54 \\
0.57 \\
0.97 \\
0.43 \\
0.50\end{array}$ & $\begin{array}{l}4 \\
4 \\
5 \\
5 \\
5 \\
4 \\
3 \\
5\end{array}$ & $\begin{array}{l}0.3 \% \\
0.3 \% \\
0.3 \% \\
0.3 \% \\
0.3 \% \\
0.3 \% \\
0.2 \% \\
0.4 \%\end{array}$ \\
\hline $\begin{array}{l}\text { Doc-I } \\
\& \\
\text { Doc-II } \\
\text { Public }\end{array}$ & $\begin{array}{l}\text { bio } \\
\text { chem } \\
\text { Eng } \\
\text { hist } \\
\text { math } \\
\text { polsci } \\
\text { soc } \\
\text { psych }\end{array}$ & $\begin{array}{r}1197 \\
912 \\
1824 \\
1026 \\
1425 \\
855 \\
798 \\
1311\end{array}$ & $\begin{array}{l}1.8 \% \\
1.4 \% \\
2.8 \% \\
1.6 \% \\
2.2 \% \\
1.3 \% \\
1.2 \% \\
2.0 \%\end{array}$ & $\begin{array}{l}54 \\
55 \\
55 \\
39 \\
57 \\
33 \\
36 \\
47\end{array}$ & $\begin{array}{l}3.6 \% \\
3.7 \% \\
3.7 \% \\
2.6 \% \\
3.8 \% \\
2.2 \% \\
2.4 \% \\
3.1 \%\end{array}$ & $\begin{array}{l}0.51 \\
0.38 \\
0.76 \\
0.61 \\
0.58 \\
0.60 \\
0.51 \\
0.64\end{array}$ & $\begin{array}{l}28 \\
21 \\
42 \\
24 \\
33 \\
20 \\
18 \\
30\end{array}$ & $\begin{array}{l}1.8 \% \\
1.4 \% \\
2.8 \% \\
1.6 \% \\
2.2 \% \\
1.3 \% \\
1.2 \% \\
2.0 \% \\
\text { ntinued) }\end{array}$ \\
\hline
\end{tabular}


TABLE 5. (Continued)

\begin{tabular}{|c|c|c|c|c|c|c|c|c|}
\hline \multirow{2}{*}{$\begin{array}{l}\text { Carnegie } \\
\text { Type }\end{array}$} & \multirow[b]{2}{*}{ Discipline } & \multicolumn{2}{|c|}{$\begin{array}{l}\text { Estimated } \\
1969 \text { Faculty }\end{array}$} & \multicolumn{2}{|c|}{$\begin{array}{c}1980 \text { UCLA } \\
\text { Subsample }\end{array}$} & \multirow[b]{2}{*}{ Weight } & \multicolumn{2}{|c|}{$\begin{array}{c}\text { Weighted } \\
1980 \text { Subsample }\end{array}$} \\
\hline & & $N$ & $\%$ & $N$ & $\%$ & & $N$ & $\%$ \\
\hline Doc-I & bio & 348 & $0.5 \%$ & 14 & $0.9 \%$ & 0.57 & 8 & $0.5 \%$ \\
\hline$\&$ & chem & 377 & $0.6 \%$ & 14 & $0.9 \%$ & 0.62 & 9 & $0.6 \%$ \\
\hline Doc-II & Eng & 580 & $0.9 \%$ & 14 & $0.9 \%$ & 0.95 & 13 & $0.9 \%$ \\
\hline \multirow[t]{5}{*}{ Private } & hist & 319 & $0.5 \%$ & 16 & $1.1 \%$ & 0.46 & 7 & $0.5 \%$ \\
\hline & math & 464 & $0.7 \%$ & 18 & $1.2 \%$ & 0.59 & 11 & $0.7 \%$ \\
\hline & polsci & 319 & $0.5 \%$ & 3 & $0.2 \%$ & 2.45 & 7 & $0.5 \%$ \\
\hline & soc & 261 & $0.4 \%$ & 7 & $0.5 \%$ & 0.86 & 6 & $0.4 \%$ \\
\hline & psych & 377 & $0.6 \%$ & 9 & $0.6 \%$ & 0.96 & 9 & $0.6 \%$ \\
\hline Comp-I & bio & 3500 & $5.4 \%$ & 28 & $1.9 \%$ & 2.88 & 81 & $5.4 \%$ \\
\hline \multirow[t]{7}{*}{ Public } & chem & 2250 & $3.5 \%$ & 17 & $1.1 \%$ & 3.05 & 52 & $3.5 \%$ \\
\hline & Eng & 5250 & $8.1 \%$ & 46 & $3.1 \%$ & 2.63 & 121 & $8.1 \%$ \\
\hline & hist & 3000 & $4.6 \%$ & 50 & $3.3 \%$ & 1.38 & 69 & $4.6 \%$ \\
\hline & math & 3500 & $5.4 \%$ & 38 & $2.5 \%$ & 2.12 & 81 & $5.4 \%$ \\
\hline & polsci & 1750 & $2.7 \%$ & 18 & $1.2 \%$ & 2.24 & 40 & $2.7 \%$ \\
\hline & soc & 2000 & $3.1 \%$ & 24 & $1.6 \%$ & 1.92 & 46 & $3.1 \%$ \\
\hline & psych & 3500 & $5.4 \%$ & 44 & $2.9 \%$ & 1.83 & 81 & $5.4 \%$ \\
\hline Comp-I & bio & 917 & $1.4 \%$ & 6 & $0.4 \%$ & 3.52 & 21 & $1.4 \%$ \\
\hline \multirow[t]{9}{*}{ Private } & chem & 786 & $1.2 \%$ & 11 & $0.7 \%$ & 1.64 & 18 & $1.2 \%$ \\
\hline & Eng & 1441 & $2.2 \%$ & 20 & $1.3 \%$ & 1.66 & 33 & $2.2 \%$ \\
\hline & hist & 786 & $1.2 \%$ & 6 & $0.4 \%$ & 3.01 & 18 & $1.2 \%$ \\
\hline & math & 1048 & $1.6 \%$ & 16 & $1.1 \%$ & 1.51 & 24 & $1.6 \%$ \\
\hline & polsci & 524 & $0.8 \%$ & 10 & $0.7 \%$ & 1.21 & 12 & $0.8 \%$ \\
\hline & soc & 524 & $0.8 \%$ & 8 & $0.5 \%$ & 1.51 & 12 & $0.8 \%$ \\
\hline & psych & 786 & $1.2 \%$ & 7 & $0.5 \%$ & 2.58 & 18 & $1.2 \%$ \\
\hline & Grand & & & & & & & \\
\hline & Total & 64882 & $100 \%$ & 1493 & $100 \%$ & & 1493 & $100 \%$ \\
\hline
\end{tabular}

Sources: Estimated faculty based on 1981-82 data reported in the American Universities and Colleges directory (1983). 
TABLE 6. 1988 University of Michigan Weight

\begin{tabular}{|c|c|c|c|c|c|c|c|c|}
\hline \multirow{2}{*}{$\begin{array}{l}\text { Carnegie } \\
\text { Type }\end{array}$} & \multirow[b]{2}{*}{ Discipline } & \multicolumn{2}{|c|}{$\begin{array}{c}\text { Estimated } \\
1988 \text { Faculty }\end{array}$} & \multicolumn{2}{|c|}{$\begin{array}{l}1988 \text { Michigan } \\
\text { Subsample }\end{array}$} & \multirow[b]{2}{*}{ Weight } & \multicolumn{2}{|c|}{$\begin{array}{c}\text { Weighted } \\
1988 \text { Subsample }\end{array}$} \\
\hline & & $N$ & $\%$ & $N$ & $\%$ & & $N$ & $\%$ \\
\hline $\begin{array}{l}\text { Res-I } \\
\text { Public }\end{array}$ & $\begin{array}{l}\text { bio } \\
\text { chem } \\
\text { Eng } \\
\text { hist } \\
\text { math } \\
\text { polsci } \\
\text { soc } \\
\text { psych }\end{array}$ & $\begin{array}{r}638 \\
870 \\
1566 \\
928 \\
1218 \\
725 \\
580 \\
957\end{array}$ & $\begin{array}{l}0.9 \% \\
1.2 \% \\
2.2 \% \\
1.3 \% \\
1.7 \% \\
1.0 \% \\
0.8 \% \\
1.4 \%\end{array}$ & $\begin{array}{l}56 \\
48 \\
64 \\
59 \\
46 \\
49 \\
53 \\
63\end{array}$ & $\begin{array}{l}2.3 \% \\
2.0 \% \\
2.7 \% \\
2.5 \% \\
1.9 \% \\
2.1 \% \\
2.2 \% \\
2.6 \%\end{array}$ & $\begin{array}{l}0.38 \\
0.61 \\
0.82 \\
0.53 \\
0.89 \\
0.50 \\
0.37 \\
0.51\end{array}$ & $\begin{array}{l}21 \\
29 \\
53 \\
31 \\
41 \\
24 \\
20 \\
32\end{array}$ & $\begin{array}{l}0.9 \% \\
1.2 \% \\
2.2 \% \\
1.3 \% \\
1.7 \% \\
1.0 \% \\
9.8 \% \\
1.4 \%\end{array}$ \\
\hline $\begin{array}{l}\text { Res-I } \\
\text { Private }\end{array}$ & $\begin{array}{l}\text { bio } \\
\text { chem } \\
\text { Eng } \\
\text { hist } \\
\text { math } \\
\text { polsci } \\
\text { soc } \\
\text { psych }\end{array}$ & $\begin{array}{l}572 \\
484 \\
550 \\
528 \\
572 \\
418 \\
286 \\
506\end{array}$ & $\begin{array}{l}0.8 \% \\
0.7 \% \\
0.8 \% \\
0.7 \% \\
0.8 \% \\
0.6 \% \\
0.4 \% \\
0.7 \%\end{array}$ & $\begin{array}{r}25 \\
24 \\
21 \\
17 \\
14 \\
15 \\
9 \\
16\end{array}$ & $\begin{array}{l}1.0 \% \\
1.0 \% \\
0.9 \% \\
0.7 \% \\
0.6 \% \\
0.6 \% \\
0.4 \% \\
0.7 \%\end{array}$ & $\begin{array}{l}0.77 \\
0.68 \\
0.88 \\
1.05 \\
1.38 \\
0.94 \\
1.07 \\
1.06\end{array}$ & $\begin{array}{l}19 \\
16 \\
19 \\
18 \\
19 \\
14 \\
10 \\
17\end{array}$ & $\begin{array}{l}0.8 \% \\
0.7 \% \\
0.8 \% \\
0.7 \% \\
0.8 \% \\
0.6 \% \\
0.4 \% \\
0.7 \%\end{array}$ \\
\hline $\begin{array}{l}\text { Res-II } \\
\text { Public }\end{array}$ & $\begin{array}{l}\text { bio } \\
\text { chem } \\
\text { Eng } \\
\text { hist } \\
\text { math } \\
\text { polsci } \\
\text { soc } \\
\text { psych }\end{array}$ & $\begin{array}{r}726 \\
792 \\
1287 \\
726 \\
1056 \\
594 \\
528 \\
759\end{array}$ & $\begin{array}{l}1.0 \% \\
1.1 \% \\
1.8 \% \\
1.0 \% \\
1.5 \% \\
0.8 \% \\
0.7 \% \\
1.1 \%\end{array}$ & $\begin{array}{l}23 \\
22 \\
32 \\
23 \\
10 \\
20 \\
27 \\
32\end{array}$ & $\begin{array}{l}1.0 \% \\
0.9 \% \\
1.3 \% \\
1.0 \% \\
0.4 \% \\
0.8 \% \\
1.1 \% \\
1.3 \%\end{array}$ & $\begin{array}{l}1.06 \\
1.21 \\
1.35 \\
1.06 \\
3.55 \\
1.00 \\
0.66 \\
0.80\end{array}$ & $\begin{array}{l}24 \\
27 \\
43 \\
24 \\
36 \\
20 \\
18 \\
26\end{array}$ & $\begin{array}{l}1.0 \% \\
1.1 \% \\
1.8 \% \\
1.0 \% \\
1.5 \% \\
0.8 \% \\
0.7 \% \\
1.1 \%\end{array}$ \\
\hline $\begin{array}{l}\text { Res-II } \\
\text { Private }\end{array}$ & $\begin{array}{l}\text { bio } \\
\text { chem } \\
\text { Eng } \\
\text { hist } \\
\text { math } \\
\text { polsci } \\
\text { soc } \\
\text { psych }\end{array}$ & $\begin{array}{r}168 \\
168 \\
266 \\
210 \\
210 \\
140 \\
98 \\
210\end{array}$ & $\begin{array}{l}0.2 \% \\
0.2 \% \\
0.4 \% \\
0.3 \% \\
0.3 \% \\
0.2 \% \\
0.1 \% \\
0.3 \%\end{array}$ & $\begin{array}{r}10 \\
8 \\
10 \\
4 \\
4 \\
7 \\
5 \\
4\end{array}$ & $\begin{array}{l}0.4 \% \\
0.3 \% \\
0.4 \% \\
0.2 \% \\
0.2 \% \\
0.3 \% \\
0.2 \% \\
0.2 \%\end{array}$ & $\begin{array}{l}0.57 \\
0.71 \\
0.90 \\
1.77 \\
1.77 \\
0.67 \\
0.66 \\
1.77\end{array}$ & $\begin{array}{l}6 \\
6 \\
9 \\
7 \\
7 \\
5 \\
3 \\
7\end{array}$ & $\begin{array}{l}0.2 \% \\
0.2 \% \\
0.4 \% \\
0.3 \% \\
0.3 \% \\
0.2 \% \\
0.1 \% \\
0.3 \%\end{array}$ \\
\hline $\begin{array}{l}\text { Doc-I } \\
\& \\
\text { Doc-II } \\
\text { Public }\end{array}$ & $\begin{array}{l}\text { bio } \\
\text { chem } \\
\text { Eng } \\
\text { hist } \\
\text { math } \\
\text { polsci } \\
\text { soc } \\
\text { psych }\end{array}$ & $\begin{array}{r}1197 \\
945 \\
1764 \\
1134 \\
1512 \\
756 \\
819 \\
1197\end{array}$ & $\begin{array}{l}1.7 \% \\
1.3 \% \\
2.5 \% \\
1.6 \% \\
2.1 \% \\
1.1 \% \\
1.2 \% \\
1.7 \%\end{array}$ & $\begin{array}{l}45 \\
48 \\
76 \\
54 \\
70 \\
35 \\
32 \\
60\end{array}$ & $\begin{array}{l}1.9 \% \\
2.0 \% \\
3.2 \% \\
2.3 \% \\
2.9 \% \\
1.5 \% \\
1.3 \% \\
2.5 \%\end{array}$ & $\begin{array}{l}0.90 \\
0.66 \\
0.78 \\
0.71 \\
0.73 \\
0.73 \\
0.86 \\
0.67\end{array}$ & $\begin{array}{l}40 \\
32 \\
59 \\
38 \\
51 \\
25 \\
28 \\
40\end{array}$ & $\begin{array}{l}1.7 \% \\
1.3 \% \\
2.5 \% \\
1.6 \% \\
2.1 \% \\
1.1 \% \\
1.2 \% \\
1.7 \% \\
\text { intinued) }\end{array}$ \\
\hline
\end{tabular}


TABLE 6. (Continued)

\begin{tabular}{|c|c|c|c|c|c|c|c|c|}
\hline \multirow{2}{*}{$\begin{array}{l}\text { Carnegie } \\
\text { Type }\end{array}$} & \multirow[b]{2}{*}{ Discipline } & \multicolumn{2}{|c|}{$\begin{array}{l}\text { Estimated } \\
1988 \text { Faculty }\end{array}$} & \multicolumn{2}{|c|}{$\begin{array}{l}198 \text { Michigan } \\
\text { Subsample }\end{array}$} & \multirow[b]{2}{*}{ Weight } & \multicolumn{2}{|c|}{$\begin{array}{c}\text { Weighted } \\
\text { 1988 Subsample }\end{array}$} \\
\hline & & $N$ & $\%$ & $N$ & $\%$ & & $N$ & $\%$ \\
\hline Doc-I & bio & 611 & $0.9 \%$ & 21 & $0.9 \%$ & 0.98 & 21 & $0.9 \%$ \\
\hline$\&$ & chem & 517 & $0.7 \%$ & 16 & $0.7 \%$ & 1.09 & 17 & $0.7 \%$ \\
\hline Doc-II & Eng & 846 & $1.2 \%$ & 39 & $1.6 \%$ & 0.73 & 28 & $1.2 \%$ \\
\hline \multirow[t]{5}{*}{ Private } & hist & 517 & $0.7 \%$ & 30 & $1.3 \%$ & 0.58 & 17 & $0.7 \%$ \\
\hline & math & 799 & $1.1 \%$ & 18 & $0.8 \%$ & 1.49 & 27 & $1.1 \%$ \\
\hline & polsci & 470 & $0.7 \%$ & 17 & $0.7 \%$ & 0.93 & 16 & $0.7 \%$ \\
\hline & soc & 329 & $0.5 \%$ & 18 & $0.8 \%$ & 0.62 & 11 & $0.5 \%$ \\
\hline & psych & 611 & $0.9 \%$ & 28 & $1.2 \%$ & 0.73 & 21 & $0.9 \%$ \\
\hline Comp-I & bio & 4275 & $6.0 \%$ & 112 & $4.7 \%$ & 1.28 & 144 & $6.0 \%$ \\
\hline \multirow[t]{7}{*}{ Public } & chem & 2565 & $3.6 \%$ & 80 & $3.4 \%$ & 1.08 & 86 & $3.6 \%$ \\
\hline & Eng & 5985 & $8.5 \%$ & 139 & $5.8 \%$ & 1.45 & 201 & $8.5 \%$ \\
\hline & hist & 3420 & $4.8 \%$ & 82 & $3.4 \%$ & 1.40 & 115 & $4.8 \%$ \\
\hline & math & 4845 & $6.8 \%$ & 102 & $4.3 \%$ & 1.60 & 163 & $6.8 \%$ \\
\hline & polsci & 2280 & $3.2 \%$ & 59 & $2.5 \%$ & 1.30 & 77 & $3.2 \%$ \\
\hline & soc & 2280 & $3.2 \%$ & 59 & $2.5 \%$ & 1.30 & 77 & $3.2 \%$ \\
\hline & psych & 3705 & $5.2 \%$ & 106 & $4.4 \%$ & 1.18 & 125 & $5.2 \%$ \\
\hline Comp-1 & bio & 852 & $1.2 \%$ & 22 & $0.9 \%$ & 1.30 & 29 & $1.2 \%$ \\
\hline \multirow[t]{9}{*}{ Private } & chem & 710 & $1.0 \%$ & 21 & $0.9 \%$ & 1.14 & 24 & $1.0 \%$ \\
\hline & Eng & 1704 & $2.4 \%$ & 56 & $2.3 \%$ & 1.02 & 57 & $2.4 \%$ \\
\hline & hist & 994 & $1.4 \%$ & 22 & $0.9 \%$ & 1.52 & 33 & $1.4 \%$ \\
\hline & math & 1420 & $2.0 \%$ & 37 & $1.6 \%$ & 1.29 & 48 & $2.0 \%$ \\
\hline & polsci & 710 & $1.0 \%$ & 21 & $0.9 \%$ & 1.14 & 24 & $1.0 \%$ \\
\hline & soc & 710 & $1.0 \%$ & 15 & $0.6 \%$ & 1.59 & 24 & $1.0 \%$ \\
\hline & psych & 994 & $1.4 \%$ & 23 & $1.0 \%$ & 1.45 & 33 & $1.4 \%$ \\
\hline & Grand & & & & & & & \\
\hline & Total & 70809 & $100 \%$ & 2383 & $100 \%$ & & 2383 & $100 \%$ \\
\hline
\end{tabular}

Sources: Estimated faculty based on 1985-86 data reported in the American Universities and Colleges directory (1987).

\section{NOTES}

1. That they do not agree is not surprising since there is not an accepted common definition for full-time faculty (e.g., $100 \%$, greater than $50 \%$, a dean or president with a faculty title but doing no faculty work, etc.).

2. The national surveys measure a variety of faculty attitudes regarding specific concerns, such as "red-baiting during the 1950s (Lazarsfeld and Theilens, 1958), faculty unionization (Ladd and Lipset, 1975, 1977), minority access (Astin, 1982), and the quality of teaching (Camegie, 1989a).

3. Studies that have relied on data from two or more of the national surveys include Astin (1984); Bentley and Blackburn (in press); Blackburn, Lawrence, Bieber, and Yoon (1988); and Carnegie (1989a). 
4. Bieber's sample included three (biology, English, psychology) of the eight disciplines. Also Bieber's base year was 1970-71 as compared to $1966-67$ for this study.

5. Smaller public Comp-I institutions were less likely to be included in the random sample because these institutions were more likely to have incomplete faculty data and thereby be excluded.

6. Most of the national surveys identify faculty from 60 to 80 disciplines/fields. However, the categories change somewhat from survey to survey.

\section{REFERENCES}

Allison, Paul D., and Stewart, John A. (1974). Productivity differences among scientists: Evidence for accumulative advantage. American Sociological Review 39: 596-606.

American Council on Education (1987). Fact Book. Washington, DC: American Council on Education.

American Universities and Colleges (1968). 10th edition. Washington, DC: American Council on Education.

American Universities and Colleges (1983). Washington, DC: American Council on Education.

American Universities and Colleges (1987). Washington, DC: American Council on Education.

Astin, Alexander W. (1982). Minorities in American Higher Education. San Francisco, CA: Jossey-Bass.

Astin, Helen S. (1978). Factors affecting women's scholarly productivity. In H. S. Astin and W. Z. Hirsch (eds.), Essays in Honor of Rosemary Park (pp. 133-157). New York: Praeger.

Bayer, Alan E. (1970). College and University Faculty: A Statistical Description (vol. 5, no. 5). Washington, DC: American Council on Education.

Bentley, Richard J., and Blackburn, Robert T. (in press). Changes in academic research performance over time: A study of institutional accumulative advantage. Research in Higher Education.

Berelson, Bernard (1960). Graduate Education in the United States. New York: McGraw-Hill.

Bieber, Jeffrey P. (1990). Faculty research productivity 1972-1988: Development of constant units of measurement. Doctoral dissertation, University of Michigan.

Biglan, Anthony (1973). The characteristics of subject matter in different academic areas. Journal of Applied Psychology 57(3): 195-203.

Blackburn, Robert T., Boberg, Alice, O'Connell, Coleman, and Pellino, Glenn R. (1980). Project for Faculty Development Program Evaluation. Ann Arbor, MI: Center for the Study of Higher Education, University of Michigan.

Blackburn, Robert T., and Lawrence, Janet H. (1989). Faculty at Work: Final Report of the National Survey. Ann Arbor, MI: National Center for Research to Improve Postsecondary Teaching and Learning, University of Michigan.

Blackburn, Robert T., and Christopher, Mackie (1990). Faculty attitude and behavior reliabilities on the University of Michigan 1988 national faculty survey. Unpublished manuscript.

Blackburn, Robert T., Lawrence, Janet H., Bieber, Jeffrey P., and Yoon, Kwang (1988). Changes in the faculty work environment 1968-1984. Paper presented at the annual meeting of the American Education Research Association, New Orleans, LA. 
Bowen, Howard R., and Schuster, Jack H. (1986). American Professors: A National Resource Imperiled. New York: Oxford Press.

Carnegie Commission on Higher Education (1973). A Classification of Institutions of Higher Education.

Carnegie Council on Policy Studies in Higher Education (1976). A Classification of Institutions of Higher Education (revised edition).

Carnegie Foundation for the Advancement of Teaching (1984). Technical Report: 1984 Carnegie Foundation National Surveys of Higher Education. Princeton, NJ: Opinion Research Corporation.

Carnegie Foundation for the Advancement of Teaching (1987, July 8). Carnegie foundation's classifications of more than 3,300 institutions of higher education. Chronicle of Higher Education, pp. 22-26, 28-30.

Carnegie Foundation for the Advancement of Teaching (1989a). A Technical Report: The Condition of the Professoriate. Princeton, NJ: Princeton University Press.

Carnegie Foundation for the Advancement of Teaching (1989b). Survey Among College and University Faculty. Princeton, NJ: The Carnegie Foundation for the Advancement of Teaching.

Clark, Mary J., and Centra, John A. (1985). Influences on the career accomplishments of Ph.D.'s Research in Higher Education 23(3): 256-269.

Drew, David E., and Tronvig, Jill A. (1988). Assessing the Quality of National Data About Academic Scientists. Claremont, CA.: Claremont Graduate School.

Higher Education Research Institute (n.d.). Technical report to 1980 national survey (exact title unknown). Los Angeles, CA.: Higher Education Research Institute, University of California, Los Angeles.

Ladd, Everett C., and Lipset, Seymour M. (1975). Technical Report: 1975 Survey of the American Professoriate. Storrs, CT: University of Connecticut.

Ladd, Everett C., and Lipset, Seymour M. (1977). Technical Report: 1977 Survey of the American Professoriate. Storrs, CT: University of Connecticut.

Lazarsfeld, Paul F., and Theilens, Wagner (1958). The Academic Mind: Social Scientists in a Time of Crisis. Glencoe, IL: Free Press.

Long, John S. (December 1978). Productivity and academic position in the scientific career. American Sociological Review 43: 889-908.

Long, John S., and McGinnis, Robert (August 1981). Organizational context and scientific productivity. American Sociological Review 46: 422-442.

Morton, Herbert S., and Price, Anne J. (1986). The ACLS survey of scholars: Views on publications, computers, libraries. Scholarly Communication 5: 1-16. Washington, DC: American Council of Learned Societies.

National Center for Educational Statistics (1989). NCES-2000. Washington, DC: Department of Education.

Office of Educational Research and Improvement (1990). Survey Report: 1988 National Survey of Postsecondary Faculty. NCES 90-365 Washington, DC: U.S. Department of Education.

Parsons, Talcott, and Platt, Gerald M. (1968), The American Academic Profession: A Pilot Study. Cambridge, MA: Harvard University Press.

Roizen, Judith, Fulton, Oliver, and Trow, Martin A. (1978). Technical Report: 1975 Carnegie Council National Surveys of Higher Education. Berkeley, CA: Center for Studies in Higher Education, University of California, Berkeley.

Shyrock, R. H. (1959). The University of Pennsylvania Faculty: A Study of American Higher Education. Philadelphia, PA: University of Pennsylvania Press.

Trow, Martin A. (1975). Technical report on the 1969 Carnegie Commission survey of 
faculty and student opinion. In Martin A. Trow (ed.), Teachers and Students. Berkeley, CA: The Carnegie Foundation for the Advancement of Teaching.

Wanner, Richard A., Lewis, Lionel S., and Gregorio, David I. (October 1981). Research productivity in academia: A comparative study of the sciences, social sciences, and humanities. Sociology of Education 54: 238-253.

Wilson, Logan (1942). The Academic Man. New York: Oxford University Press.

Received August 21, 1990 